\title{
TU/e EmonOWEN

\section{Constrained iterative feedback tuning for robust control of a wafer stage system}

\section{Citation for published version (APA):}

Heertjes, M. F., Velden, van der, B. J. C. H., \& Oomen, T. A. E. (2016). Constrained iterative feedback tuning for robust control of a wafer stage system. IEEE Transactions on Control Systems Technology, 24(1), 56-66. https://doi.org/10.1109/TCST.2015.2418311

\section{Document license:}

TAVERNE

DOI:

10.1109/TCST.2015.2418311

Document status and date:

Published: 01/01/2016

\section{Document Version:}

Publisher's PDF, also known as Version of Record (includes final page, issue and volume numbers)

\section{Please check the document version of this publication:}

- A submitted manuscript is the version of the article upon submission and before peer-review. There can be important differences between the submitted version and the official published version of record. People interested in the research are advised to contact the author for the final version of the publication, or visit the $\mathrm{DOI}$ to the publisher's website.

- The final author version and the galley proof are versions of the publication after peer review.

- The final published version features the final layout of the paper including the volume, issue and page numbers.

Link to publication

\section{General rights}

Copyright and moral rights for the publications made accessible in the public portal are retained by the authors and/or other copyright owners and it is a condition of accessing publications that users recognise and abide by the legal requirements associated with these rights.

- Users may download and print one copy of any publication from the public portal for the purpose of private study or research.

- You may not further distribute the material or use it for any profit-making activity or commercial gain

- You may freely distribute the URL identifying the publication in the public portal.

If the publication is distributed under the terms of Article 25fa of the Dutch Copyright Act, indicated by the "Taverne" license above, please follow below link for the End User Agreement:

www.tue.nl/taverne

Take down policy

If you believe that this document breaches copyright please contact us at:

openaccess@tue.nl

providing details and we will investigate your claim. 


\title{
Constrained Iterative Feedback Tuning for Robust Control of a Wafer Stage System
}

\author{
Marcel F. Heertjes, Bart Van der Velden, and Tom Oomen
}

\begin{abstract}
Iterative feedback tuning (IFT) enables the data-driven tuning of controller parameters without the explicit need for a parametric model. It is known, however, that IFT can lead to nonrobust solutions. The aim of this paper is to develop an IFT approach with robustness constraints. A constrained IFT problem is formulated that is solved by introducing a penalty function. Essentially, the gradient estimates decompose into: 1) the well-known IFT gradients and 2) the gradients with respect to this penalty function. The latter are obtained through a nonparametric model of the controlled system. This guarantees robust stability while only requiring a nonparametric model. The experimental results obtained from the motion control systems of an industrial wafer scanner confirm enhanced performance with guaranteed robustness estimates.
\end{abstract}

Index Terms-Data-driven control, high-precision wafer stages, iterative feedback tuning (IFT), robust control.

\section{INTRODUCTION}

C ONTROL systems are often designed using either model-based approaches or data-based approaches. Model-based approaches refer to the system first being modeled, for example, through system identification. Modeling, however, can be a time-consuming and difficult process [19], [33]. In this regard, direct data-driven approaches (see [2] for an overview) are appealing since they avoid the need for modeling and enable the direct tuning of the controller based on measurement data. This has led to a variety of data-based control design methods including unfalsified control [38], virtual reference feedback tuning [7], and iterative feedback tuning (IFT) [15], [18]. Interestingly, in [19], it is shown that many of the data-based approaches can be interpreted as being model based, except for IFT. IFT is an iterative optimization approach that aims at obtaining unbiased

Manuscript received June 20, 2014; revised January 12, 2015; accepted March 2, 2015. Date of publication April 20, 2015; date of current version December 21, 2015. Manuscript received in final form March 23, 2015. This work was supported by the Netherlands Organization for Scientific Research and the Dutch Science Foundation Innovational Research Incentives Scheme under VENI Grant 13073, titled Precision Motion: Beyond the Nanometer. Recommended by Associate Editor N. H. El-Farra.

M. F. Heertjes is with the Department of Mechanical Engineering, Eindhoven University of Technology, Eindhoven 5600 MB, The Netherlands, and also with Mechatronics System Development, ASML, Veldhoven 5504 DR, The Netherlands (e-mail: marcel.heertjes@asml.com).

B. Van der Velden was with the Eindhoven University of Technology, Eindhoven $5600 \mathrm{MB}$, The Netherlands. He is now with TMC Group, Eindhoven 5657 DC, The Netherlands (e-mail: bart.van.der.velden@ asml.com).

T. Oomen is with the Control Systems Technology Group, Department of Mechanical Engineering, Eindhoven University of Technology, Eindhoven 5600 MB, The Netherlands (e-mail: t.e.a.oomen@tue.nl).

Color versions of one or more of the figures in this paper are available online at http://ieeexplore.ieee.org.

Digital Object Identifier 10.1109/TCST.2015.2418311 gradient estimates by conducting multiple experiments. In a gradient-based update scheme, the gradient estimates are used to compute a new set of controller parameters. The optimal set of parameters corresponds to a minimum of a performance-relevant cost function in time domain. IFT has been successfully applied in many applications, including process industry [9], [29], robotics [10], [23], [36], mechatronics [1], [28], and stage control [45]. Most of these works involve linear (time-invariant) systems. For nonlinear systems, IFT has been addressed in [11] and [40].

An important field of application where data-based controller tuning is appealing is in high-precision motion control. For feedforward control design, model-based approaches have led to limited performance enhancements due to model uncertainty [4]. This has led to the development of data-based approaches in [13], and [30] for the multivariable case, which induce significant performance enhancements. It has recently been shown in [3] that the IFT algorithm employed in [30] for feedforward control has a direct system identification interpretation. In fact, for the case of feedforward control, the IFT algorithm deals with a closed-loop identification problem, but comes at the expense of efficiency. In [3], more efficient algorithms have been proposed, rendering the use of IFT superfluous for feedforward design. Still, IFT seems appealing for feedback design. This is because of the following.

1) IFT requires no parametric system model.

2) Unlike many model-based synthesis algorithms, IFT can deal with a predefined controller structure.

3) Control performance is optimized for the disturbance situation at hand, so no disturbance model is needed.

Despite these clear advantages, a direct IFT implementation suffers from robustness issues as there are generally no guarantees that the closed-loop system remains stable during the iterations. This is evidenced by the development of IFT algorithms that take into account robust stability [5], [34], [42]. These algorithms typically use approximations of robustness measures, including the $v$-gap metric [43] and $\mathcal{H}_{\infty}$ norms of relevant closed-loop transfer functions, which may be rather conservative. Moreover, strong guarantees for robust stability are not provided.

The main contribution of this paper is a new approach for constrained IFT. To ensure robustness, a frequency-domain constraint is added to the objective function that represents the robustness objective. For the considered class of motion systems, nonparametric frequency response function models are relatively inexpensive, fast, and accurate to obtain. Although not directly usable for controller synthesis, since 

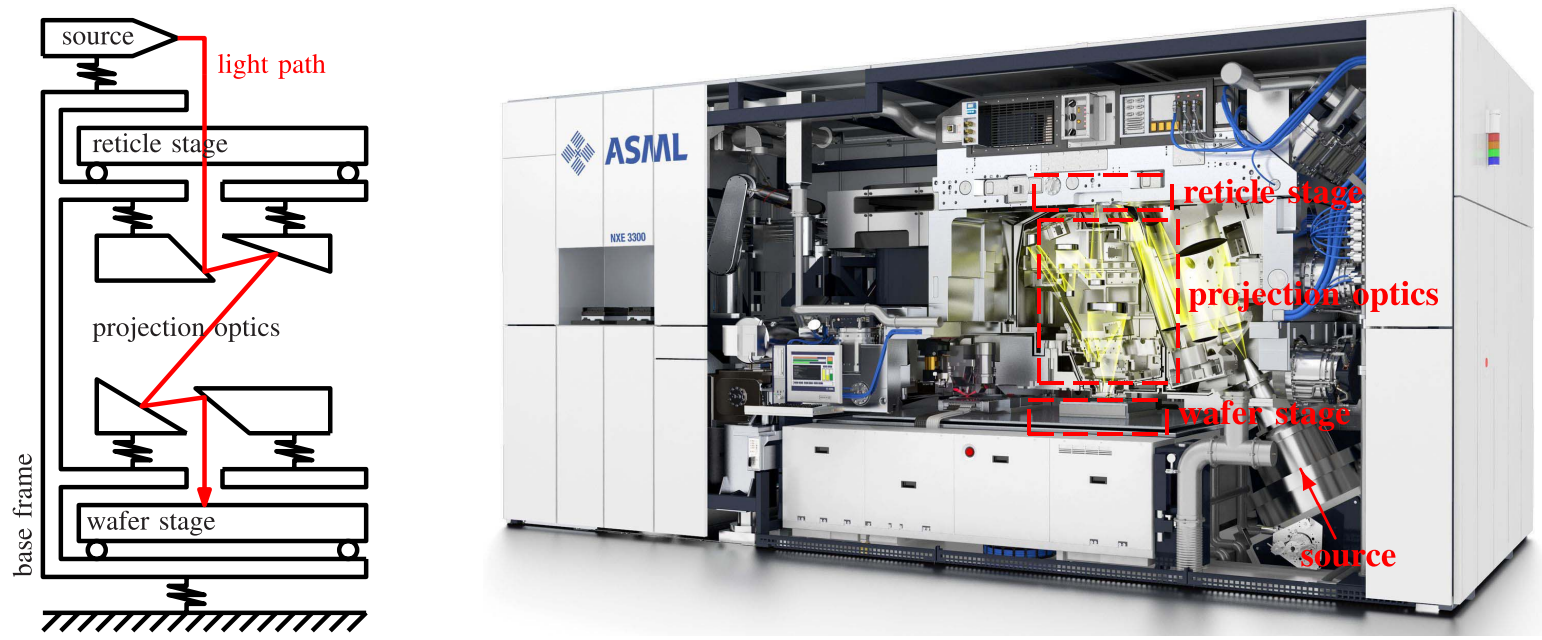

Fig. 1. Schematics and artist impression of a wafer scanner.

synthesis generally requires a parametric model, these models are well suited to evaluate robustness margins such as the modulus margin. The preliminary results of this new approach are given in [41]. This paper extends these results by thoroughly demonstrating the effectiveness of the approach on the wafer stage subsystem of a wafer scanner, i.e., a lithography machine. Apart from fine-tuning of the PID controller parameters, this also involves fine-tuning of the notch filter parameters that in [41] are fixed a priori by manual loop shaping.

The central idea in this paper is to include a constraint, e.g., the modulus margin, in the optimization criterion. By means of a penalty function, the gradient-based IFT scheme can be decomposed into: 1) the well-known IFT gradients and 2) the gradients with respect to this penalty function. This is related to [12] where an interior-point method is adopted to take into account signal constraints on the input. In our approach, the numerical differentiation is done offline based on the identified nonparametric model. During offline optimization, a modified optimal set of controller parameters is obtained that satisfies the frequency-domain robustness specifications. With this set, a new IFT experiment is performed. Given the accuracy of the nonparametric model, the experimental results demonstrate that no experiments are done that involve (significant) violations of the frequency-domain robustness specifications, i.e., no controllers are implemented that can possibly lead to unstable control design. This is important for high-precision motion systems like wafer scanners where machine damage should be avoided due to high costs.

This paper is organized as follows. In Section II, the wafer scanner system and the wafer stage subsystem are discussed. In Section III, a short review of IFT is given in the wafer stage context. In Section IV, the penalty function approach is introduced as a means to impose frequency-domain specifications and the extended optimization algorithm underlying this approach is presented. In Section V, the measurement results obtained from the considered wafer stage system are presented. In Section VI, the main conclusions are given.

\section{Wafer Scanners AND Wafer Stage Control}

Wafer scanners are lithography machines used in the process of producing integrated circuits (see [27] for the principles of lithography). In this section, the control tuning problem of wafer scanners in general and wafer stages in specific is introduced (see also [6] for an historical perspective and [33] for an overview of the present control challenges). The section is divided into five parts: 1) wafer scanners; 2) motion control context; 3) wafer stage plant description; 4) wafer stage feedback control; and 5) performance of wafer scanners and stages. This completely outlines the setting in which the IFT algorithm is implemented.

\section{A. Wafer Scanner Stages}

A schematic of a wafer scanner is shown in Fig. 1. Extreme ultraviolet light travels from a source (located outside the machine) through projection optics to the light sensitive layers of a wafer (a silicon disk of 300-mm radius). The light contains an image of the integrated circuits to be processed. The image is obtained from the reticle that is part of the reticle stage motion control system. The wafer is part of the wafer stage motion control system. During wafer scanning, i.e., the dose-controlled wafer exposure by an extreme ultraviolet light beam, both the reticle and the wafer stage systems track a series of point-to-point motions in the (scanning) $y$-direction [24]. In this paper, the focus is on the wafer stage system. Due to the close similarity of the two systems, the presented approach directly applies to the reticle stage system.

The wafer stage system consists of two modules: 1) the long-stroke module and 2) the short-stroke module. The positioning accuracy of the long-stroke module is at micrometer level. In scanning an entire wafer, the long-stroke module, which weighs $\approx 67 \mathrm{~kg}$, provides support for the short-stroke module along the different fields of the wafer; typically, 100 fields of approximately $30 \mathrm{~mm} \times 30 \mathrm{~mm}$ need to be exposed sequentially. The positioning accuracy of the short-stroke module, which weighs $\approx 18 \mathrm{~kg}$ and which supports the wafer, is at nanometer level. All fields on a wafer are scanned in the $y$-direction by conducting a series of point-topoint motions. Each point-to-point motion has an interval-the scanning interval-of constant velocity. Apart from the scanning interval, the point-to-point motion profiles include acceleration/deceleration intervals in the $x$ and $y$ directions 


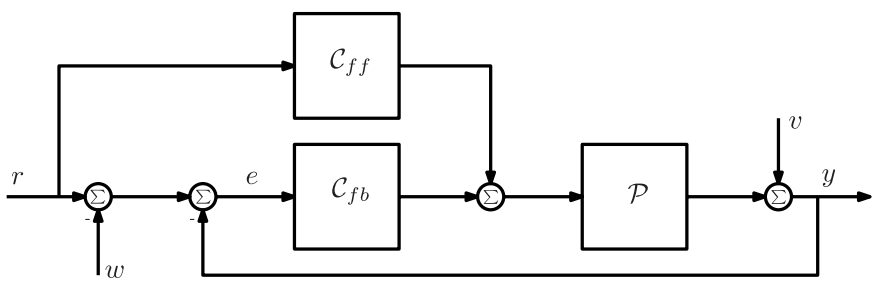

Fig. 2. Block diagram of the simplified wafer stage motion control context.

so as to enable full wafer coverage. For the purpose of positioning, the wafer stage actuator systems use voice coil motors, whereas the main sensor systems are based on interferometry. The wafer stage modules are controlled in six logical axes: $x, y, r_{z}, z, r_{x}$, and $r_{y}$, using a diagonal controller structure in combination with an actuator/sensor decoupling design. In this paper, we only consider the (scanning) $y$-direction.

\section{B. Motion Control Context}

The motion control context of a simplified wafer stage system is shown in Fig. 2. The linear time-invariant wafer stage plant $\mathcal{P}$ has output $y$, which is corrupted by the unmeasured disturbances $v$. These disturbances are assumed to have a stochastic nature and are not correlated with the other inputs $r$ and $w$. Plant $\mathcal{P}$ is assumed to be single-input single-output and controlled by a linear time-invariant feedforward controller $\mathcal{C}_{f f}$ and a feedback controller $\mathcal{C}_{f b}$. Controller $\mathcal{C}_{f b}$ is a function of the parameters $\boldsymbol{\rho}=[\boldsymbol{\rho}(1) \ldots \rho(m)]^{T}$ to be optimized, i.e., $\mathcal{C}_{f b}=\mathcal{C}_{f b}(\boldsymbol{\rho})$, with the number of parameters $m \in\{1,2, \ldots\}$. In conducting point-to-point motion, tracking performance is reflected by the closed-loop error $e=r-w-y$, i.e., the difference between the reference command $r$, the output $y$, and an auxiliary input $w$ used for the IFT experiments. This auxiliary input is explained in more detail in Section III.

Remark 1: Though $\mathcal{C}_{f b}$ and $\mathcal{C}_{f f}$ can be optimized jointly, in this paper, the feedforward controller $\mathcal{C}_{f f}$ will not be subject to (constrained) IFT optimization. Basically, $\mathcal{C}_{f f}$ poses no (robust) stability problem. Besides, for feedforward parameter tuning, which often refers to finding an acceleration term, a snap term, and a delay compensation term, more efficient algorithms are available, see for example [3] and [30].

\section{Wafer Stage Plant Description}

In Fig. 3, it can be observed by frequency response measurements that the short-stroke wafer stage plant description $\mathcal{P}$ essentially boils down to a double-integrator-based system with a mass of approximately $17.7 \mathrm{~kg}$. This follows from the $-40-\mathrm{dB} / \mathrm{dec}$ ade magnitude slope in the frequency interval between 40 and $400 \mathrm{~Hz}$ combined with a $-180^{\circ}$ phase lag. At low frequencies, a poor signal-tonoise ratio leads to a poor identification result. The main reason is that the closed-loop system identification procedure, as described in [33, Appendix A], is followed. Essentially, the frequency response function of the open-loop system is obtained by dividing the measured closed-loop process sensitivity data by the closed-loop sensitivity data. Since the
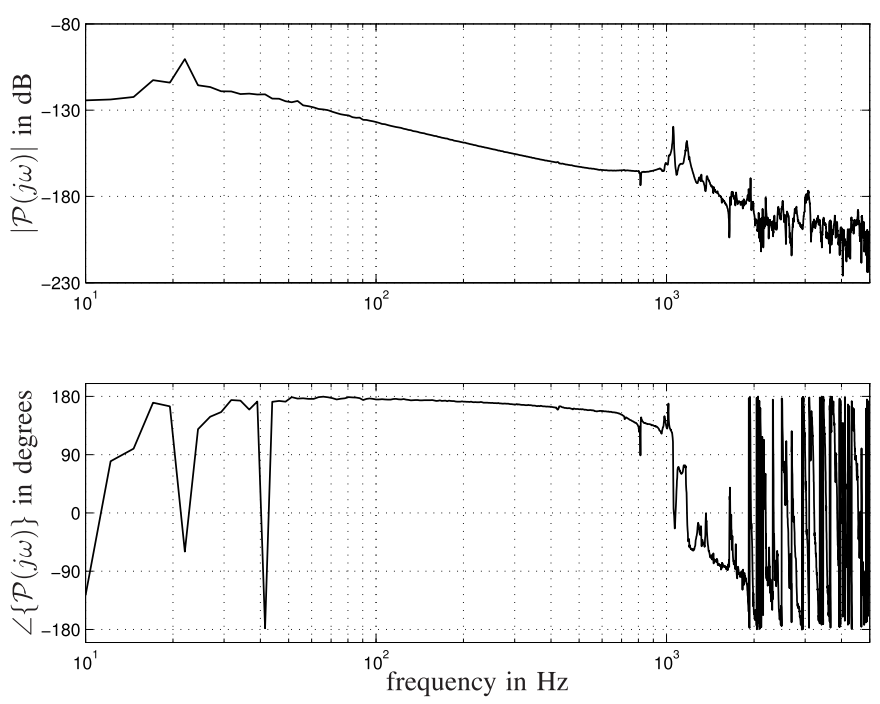

Fig. 3. Bode diagram of the short-stroke wafer stage plant $\mathcal{P}$ in the $y$-direction with measured data.

sensitivity function is typically small at low frequencies due to a high controller gain, the signal-to-noise ratio for an arbitrary and nonoptimized experiment design is poor. At high frequencies, several resonances appear that characterize the structural flexibilities of the stage system. For example, around $1 \mathrm{kHz}$, a torsion mode is responsible for the observed behavior. In the frequency response measurements, a sampling time of $T=10^{-4} \mathrm{~s}$ is used.

Remark 2: The wafer stage system is a multivariable system that through a proper choice of input/output decoupling matrices becomes diagonally dominant. Multiinput multi-output (MIMO) stage control is therefore often done with a diagonal controller structure in the 6 DOF: $x, y, r_{z}, z, r_{x}, r_{y}$. For clarity of presentation, only the scanning $y$-direction is subject to further (constrained) IFT optimization (see [16], [17], and [35] for examples of MIMO IFT and [33] and [44] for MIMO model-based counterparts).

\section{Wafer Stage Feedback Control}

The short-stroke wafer stage plant $\mathcal{P}$ in the $y$-direction is controlled by the feedback controller $\mathcal{C}_{f b}$, which (in continuous time) has the following structure:

$$
\mathcal{C}_{f b}(s)=\mathcal{C}_{\mathrm{PID}}(s) \mathcal{C}_{\mathrm{LP}}(s) \mathcal{C}_{N}(s)
$$

with $s$ the Laplace variable. The PID-controller part $\mathcal{C}_{\text {PID }}$, the low-pass part $\mathcal{C}_{\mathrm{LP}}$, and the notch part $\mathcal{C}_{N}$ are given by

$$
\begin{aligned}
\mathcal{C}_{\mathrm{PID}}(s) & =k_{p}\left(\frac{s}{\omega_{d}}+1+\frac{\omega_{i}}{s}\right) \\
\mathcal{C}_{\mathrm{LP}}(s) & =\frac{\omega_{l p}^{2}}{s^{2}+2 \zeta{ }_{l p} \omega_{l p} s+\omega_{l p}^{2}} \\
\mathcal{C}_{N}(s) & =\prod_{i=1}^{n} \mathcal{N}_{i}(s)
\end{aligned}
$$

with the notch filters

$$
\mathcal{N}_{i}(s)=\left(\frac{\omega_{p, i}}{\omega_{z, i}}\right) \frac{s^{2}+2 \zeta_{z, i} \omega_{z, i} s+\omega_{z, i}^{2}}{s^{2}+2 \zeta_{p, i} \omega_{p, i} s+\omega_{p, i}^{2}} .
$$


TABLE I

Fixed Parameters of the Wafer Stage Feedback Controller $\mathcal{C}_{f b}$

\begin{tabular}{ccccc}
\hline $\mathcal{C}_{P I D}$ & $k_{p}$ in $\mathrm{Nm}^{-1}$ & $\omega_{i}$ in $\mathrm{rad} \cdot \mathrm{s}^{-1}$ & $\omega_{d}$ in $\mathrm{rad} \cdot \mathrm{s}^{-1}$ & \\
& $1.9 \cdot 10^{7}$ & $2 \pi \cdot 80$ & $2 \pi \cdot 130$ & \\
\hline $\mathcal{C}_{L P}$ & $\omega_{l p}$ in $\mathrm{rad} \cdot \mathrm{s}^{-1}$ & $\zeta_{l p}$ & & \\
& $2 \pi \cdot 1400$ & 0.80 & & \\
\hline $\mathcal{N}_{i}$ & $\omega_{z, i}$ in rad $\cdot \mathrm{s}^{-1}$ & $\zeta_{z, i}$ & $\omega_{p, i}$ in $\mathrm{rad} \cdot \mathrm{s}^{-1}$ & $\zeta_{p, i}$ \\
1 & $2 \pi \cdot 430$ & 0.01 & $2 \pi \cdot 430$ & 0.03 \\
2 & $2 \pi \cdot 718$ & 0.01 & $2 \pi \cdot 718$ & 0.05 \\
3 & $2 \pi \cdot 812$ & 0.01 & $2 \pi \cdot 812$ & 0.03 \\
4 & $2 \pi \cdot 1100$ & 0.12 & $2 \pi \cdot 900$ & 0.20 \\
5 & $2 \pi \cdot 1950$ & 0.01 & $2 \pi \cdot 1850$ & 0.05 \\
\hline
\end{tabular}

Five notch filters $\mathcal{N}_{1}, \ldots, \mathcal{N}_{5}$ are used to: 1) deal with the plant resonances and 2) properly shape the open-loop characteristics so as to achieve high bandwidth with sufficient robustness margins. The parameters of $\mathcal{C}_{\mathrm{PID}}, \mathcal{C}_{\mathrm{LP}}$, and $\mathcal{C}_{N}$ that correspond to the default controller and that are used as starting point for (constrained) IFT are given in Table I.

Remark 3: The controller $\mathcal{C}_{f b}$ is implemented in discrete time using the $\delta$ operator [31], where $\delta=(z-1) / T$, with $z$ the forward shift operator. The reason is because of the finite (internal) floating point resolution that otherwise induces error in the computation of the poles and zeros. This especially holds true at low frequencies where these poles and zeros generally lie closer to one than to zero.

\section{E. Wafer Scanner Stage Performance}

The performance of wafer scanners is basically given by three measures: 1) overlay; 2) imaging; and 3) throughput. Overlay refers to the ability to expose one layer of the wafer atop another; in the process of making chips, typically 20-30 subsequent layers are being exposed. Imaging generally refers to image quality in terms of linewidth uniformity. Throughput refers to the number of wafers that are being exposed per hour.

Wafer stage performance is evaluated in time domain and relates to the servo error signals that are (partly) responsible for overlay, imaging, and throughput. In terms of servo errors, throughput is related to settling times prior to scanning. Overlay and imaging mainly refer to the scanning interval and are related to, respectively, a moving average $\left(\mathcal{M}_{A}\right)$ and a moving standard deviation $\left(\mathcal{M}_{\mathrm{SD}}\right)$ filter operation applied (offline) to the servo error signals. In continuous time, these operations are given by

$$
\begin{aligned}
\mathcal{M}_{A}(t) & =\frac{1}{T_{e}} \int_{t-T_{e} / 2}^{t+T_{e} / 2} e(\tau) d \tau \\
\mathcal{M}_{\mathrm{SD}}(t) & =\sqrt{\frac{1}{T_{e}} \int_{t-T_{e} / 2}^{t+T_{e} / 2}\left(e(\tau)-\mathcal{M}_{A}(\tau)\right)^{2} d \tau}
\end{aligned}
$$

with $T_{e}$ the exposure time constant defined by $T_{e}=s / v$, where $s$ represents the slit height and $v$ is the scan velocity. The $\mathcal{M}_{A}$ filter has low-pass properties, whereas the $\mathcal{M}_{\mathrm{SD}}$ filter has high-pass properties. Both filter operations are noncausal, and therefore can only be applied offline.

Having set the stage context, IFT is considered as a means to fine-tune the controller parameters $\rho$ in $\mathcal{C}_{f b}=\mathcal{C}_{f b}(\rho)$, see (1).

\section{Iterative Feedback Tuning: The Stage Context}

In discussing IFT in the stage context, this section contains two parts: 1) an IFT overview and 2) IFT experiments and the resulting unbiased gradient estimates.

\section{A. IFT Overview}

IFT [15]-[17] can be used to find the optimal set of controller parameters $\rho^{*}$ that minimizes the cost function

$$
J=\frac{1}{2 N} \mathbf{e}^{T} \mathbf{e}
$$

with the data sampled signal $\mathbf{e}=[e(1) \ldots e(N)]^{T}, \mathbf{e}=\mathbf{e}(\rho)$, and the number of samples $N>0$. Equation (5) gives rise to the following unconstrained optimization problem:

$$
\rho^{*}=\min _{\rho} J
$$

which can be solved iteratively using the Gauss-Newton algorithm

$$
\boldsymbol{\rho}_{i+1}=\boldsymbol{\rho}_{i}-\left.\gamma_{i} H^{-1} \frac{\partial J}{\partial \boldsymbol{\rho}}\right|_{\boldsymbol{\rho}=\boldsymbol{\rho}_{i}}
$$

where $0<\gamma_{i} \leq 1$ is the step size at iteration $i$, the gradient $\partial J / \partial \rho$ is given by

$$
\frac{\partial J}{\partial \boldsymbol{\rho}}=\frac{1}{N} \frac{\partial \mathbf{e}^{T}}{\partial \boldsymbol{\rho}} \mathbf{e}
$$

and an approximation of the Hessian $H=H(\rho)$ is given by

$$
H=\frac{1}{N} \frac{\partial \mathbf{e}^{T}}{\partial \boldsymbol{\rho}} \frac{\partial \mathbf{e}}{\partial \boldsymbol{\rho}} .
$$

By seeking the minimum of (5) using a gradient-based search, from (8) and (9), it is clear that finding $\rho^{*}$ in (6) with (7) requires the gradients $\partial \mathbf{e} / \partial \boldsymbol{\rho}$.

\section{B. IFT Experiments and Gradients $\partial \boldsymbol{e} / \partial \boldsymbol{\rho}$}

In the context of Fig. 2, consider the following three IFT experiments that are conducted under identical: 1) point-to-point reference $r ; 2$ ) feedforward controller $\mathcal{C}_{f f}$; and 3) feedback controller $\mathcal{C}_{f b}$ with parameters $\rho$ [15], [18].

Experiment I: The result of the first experiment with no auxiliary input, i.e., $w_{I}=0$ (the subscript indicates the experiment number), satisfies

$$
\begin{aligned}
y_{I}(\delta) & =v_{I}(\delta)+\mathcal{P}(\delta)\left(\mathcal{C}_{f f}(\delta) r(\delta)+\mathcal{C}_{f b}(\delta)\left(r(\delta)-y_{I}(\delta)\right)\right) \\
& =\mathcal{S}(\delta) v_{I}(\delta)+\mathcal{T}(\delta)\left(\frac{\mathcal{C}_{f f}(\delta)}{\mathcal{C}_{f b}(\delta)}+1\right) r(\delta)
\end{aligned}
$$

with the sensitivity function $\mathcal{S}$ and the complementary sensitivity function $\mathcal{T}$ defined in the frequency domain by

$$
\mathcal{S}(\delta)=\frac{1}{1+\mathcal{C}_{f b}(\delta) \mathcal{P}(\delta)} \quad \mathcal{T}(\delta)=\frac{\mathcal{C}_{f b}(\delta) \mathcal{P}(\delta)}{1+\mathcal{C}_{f b}(\delta) \mathcal{P}(\delta)} .
$$

From (10), it follows that:

$$
\frac{\partial y_{I}(\delta)}{\partial \boldsymbol{\rho}}=\frac{1}{\mathcal{C}_{f b}(\delta)} \frac{\partial \mathcal{C}_{f b}(\delta)}{\partial \boldsymbol{\rho}} \mathcal{T}(\delta)\left(r(\delta)-y_{I}(\delta)\right) .
$$

The purpose of Experiment I is twofold. First, the data $y_{I}$ are used (via $e_{I}=r-y_{I}$ ) in the computation of the gradients in (8) regarding the part $\mathbf{e}=\mathbf{e}_{I}$. Second, these data define the auxiliary input $w_{I I}$ for Experiment II. 
Experiment II: Define the second experiment with the auxiliary input $w_{I I}=e_{I}$, i.e., use the closed-loop error signal from the first experiment as auxiliary input to the second experiment. Note that $w_{I I}$ is a signal that is added to the unaltered setpoint signal $r$ (recall Fig. 2). In the implementation, $w_{I I}$ therefore only needs to be synchronized in time with the setpoint signal. The second experiment gives

$$
\begin{aligned}
y_{I I}(\delta)= & \mathcal{S}(\delta) v_{I I}(\delta)+\mathcal{T}(\delta)\left(\frac{\mathcal{C}_{f f}(\delta)}{\mathcal{C}_{f b}(\delta)}+1\right) r(\delta) \\
& -\mathcal{T}(\delta) e_{I}(\delta) .
\end{aligned}
$$

The purpose of Experiment II is to construct unbiased gradient estimates $\partial J / \partial \rho$ in (8) from sole data. This is done by assuring that the noise contained in $\mathbf{e}=\mathbf{e}_{I}$ (and obtained from Experiment I) does not correlate with the noise contained in the estimates of $\partial \mathbf{e} / \partial \boldsymbol{\rho}$. For this reason, the third experiment is conducted.

Experiment III: From the third experiment, which is similar to the first experiment, i.e., $w_{I I I}=0$, we obtain

$$
y_{I I I}(\delta)=\mathcal{S}(\delta) v_{I I I}(\delta)+\mathcal{T}(\delta)\left(\frac{\mathcal{C}_{f f}(\delta)}{\mathcal{C}_{f b}(\delta)}+1\right) r(\delta) .
$$

Subtracting (14) from (13) gives

$$
\begin{aligned}
& \mathcal{T}(\delta)\left(r(\delta)-y_{I}(\delta)\right) \\
& \quad=y_{I I I}(\delta)-y_{I I}(\delta)+\mathcal{S}(\delta)\left(v_{I I}(\delta)-v_{I I I}(\delta)\right) .
\end{aligned}
$$

Hence, in view of the properties of the disturbances $v$

$$
\text { est }\left\{\mathcal{T}(\delta)\left(r(\delta)-y_{I}(\delta)\right)\right\}=y_{I I I}(\delta)-y_{I I}(\delta) .
$$

This demonstrates the ability to construct unbiased gradient estimates $\partial J / \partial \rho$ in (8) from sole data, namely, the substitution of (16) into (12) gives an estimate of the gradients in (12)

$$
\text { est }\left\{\frac{\partial y_{I}(\delta)}{\partial \boldsymbol{\rho}}\right\}=\frac{1}{\mathcal{C}_{f b}(\delta)} \frac{\partial \mathcal{C}_{f b}(\delta)}{\partial \boldsymbol{\rho}}\left(y_{I I I}(\delta)-y_{I I}(\delta)\right)
$$

which is based on the measured data and known model relations regarding the controller $\mathcal{C}_{f b}(\delta)$. From (17), the step toward the gradients $\partial \mathbf{e} / \partial \boldsymbol{\rho}$ is straightforward. Note from Fig. 2 that

$$
\frac{\partial e}{\partial \rho}=-\frac{\partial y}{\partial \rho} .
$$

With (18), the gradients $\partial \mathbf{e} / \partial \boldsymbol{\rho}$ are defined in the time domain by

$$
\frac{\partial \mathbf{e}}{\partial \boldsymbol{\rho}}=-\left[\mathbf{C}_{1}\left(\mathbf{y}_{I I I}-\mathbf{y}_{I I}\right) \ldots \mathbf{C}_{m}\left(\mathbf{y}_{I I I}-\mathbf{y}_{I I}\right)\right]
$$

with $\mathbf{C}_{1}=\mathbf{C}(\rho), \ldots, \mathbf{C}_{m}=\mathbf{C}_{m}(\boldsymbol{\rho})$ the Toeplitz matrices containing the impulse responses of the (stable) filters

$$
\mathcal{C}_{1}(\delta)=\frac{1}{\mathcal{C}_{f b}(\delta)} \frac{\partial \mathcal{C}_{f b}(\delta)}{\partial \rho(1)} \ldots \mathcal{C}_{m}(\delta)=\frac{1}{\mathcal{C}_{f b}(\delta)} \frac{\partial \mathcal{C}_{f b}(\delta)}{\partial \rho(m)}
$$

and the data-sampled signals $\mathbf{y}_{I I}=\left[y_{I I}(1) \ldots y_{I I}(N)\right]^{T}$ and $\mathbf{y}_{I I I}=\left[y_{I I I}(1) \ldots y_{I I I}(N)\right]^{T}$. In summary, the data $e_{I}=r-y_{I}$ from Experiment I are used: 1) in the computation of the gradients in (8) regarding the part $\mathbf{e}=\mathbf{e}_{I}$ and 2) to define the auxiliary input $w_{I I}$ for Experiment II. The gradient estimates in (17) are obtained from the data of Experiments II and III, i.e., in view of the uncorrelated disturbances $v_{I I}$ and $v_{I I I}$. Since $v_{I I}$ and $v_{I I I}$ in $\partial \mathbf{e} / \partial \rho$ do not correlate with $v_{I}$ in $\mathbf{e}, \partial J / \partial \rho$ in (8) becomes unbiased. This would not be the case if, instead of Experiment III, the data from (identical) Experiment I were used. Note that the approximate Hessian matrix $H$ in (9) may be biased. The unbiased estimates of the Hessian are proposed in [40].

Despite the clear advantages of having unbiased gradient estimates without the use of parametric models, IFT suffers from robustness issues as there are no guarantees that the closed-loop system remains stable during the iterations. In fact, the optimized controller is often close to instability and the danger of closed-loop instability during the iterations may be high (see also [8] for an interesting explanation). Also, strong guarantees for robust stability are not provided. To ensure robustness, a frequency-domain constraint is added to the objective function in (5) that represents the robustness objective. This will be referred to as constrained IFT.

\section{Constrained Iterative Feedback Tuning}

In this section, a new constrained IFT approach is presented as a means to impose frequency-domain specifications, e.g., the modulus margin, on the closed-loop stage system. The section is divided into four parts: 1) frequency-domain constraints; 2) unconstrained optimization problem formulation; 3) constrained IFT algorithm (this includes an extra iteration loop to penalize violations of the robustness specifications); and 4) computation of the gradients (with respect to the penalty function). The outcome of the section is an IFT method that ensures robustness.

\section{A. Frequency-Domain Constraints}

Constrained IFT will be used to find the optimal set of controller parameters $\rho^{*}$ that minimizes the cost function $J=J(\rho)$ under the constraint

$$
g \leq 0
$$

with $g=g(\rho)$ defined by

$$
g(\boldsymbol{\rho})=\max _{\omega}\left(|\mathcal{S}(\omega, \boldsymbol{\rho})|-\mathcal{S}_{b}(\omega)\right)
$$

where $\mathcal{S}=\mathcal{S}(\omega, \boldsymbol{\rho})$ is the closed-loop sensitivity function (11), and $\mathcal{S}_{b}=\mathcal{S}_{b}(\omega)>0$ is a frequency-dependent function that for each frequency, $\omega$ specifies the amplitude constraints imposed on $\mathcal{S}$. Note that the particular choice of $g$ in (22) is made to facilitate the exposition. The theory that is presented is general and can be applied to more arbitrary choices of $g$.

\section{B. Unconstrained Optimization Problem Formulation}

To transform the above-described constrained optimization problem into an unconstrained optimization problem formulation similar to (6), the cost function in (5) is extended with a penalty $J_{g}=J_{g}(\omega, \rho)$ that is based on the constraint function in (22), or

$$
J=\underbrace{\frac{1}{2 N} \mathbf{e}^{T} \mathbf{e}}_{J_{e}}+\alpha \underbrace{\frac{1}{2} \phi(g) g^{2}}_{J_{g}}
$$


with $\alpha>0$ a scaling factor and $\phi$ a piecewise linear function

$$
\phi(g)= \begin{cases}0, & \text { if } g \leq 0 \\ 1, & \text { otherwise. }\end{cases}
$$

This unconstrained optimization problem can be solved iteratively using the Gauss-Newton algorithm in (7). Different from (8), however, the gradient $\partial J / \partial \rho$ is given by

$$
\begin{aligned}
\frac{\partial J}{\partial \boldsymbol{\rho}} & =\frac{\partial J_{e}}{\partial \boldsymbol{\rho}}+\alpha \frac{\partial J_{g}}{\partial \boldsymbol{\rho}} \\
& =\frac{1}{N} \frac{\partial \mathbf{e}^{T}}{\partial \boldsymbol{\rho}} \mathbf{e}+\alpha \phi(g) g(\underbrace{\frac{\partial \phi(g)}{\partial g} \frac{\partial g}{\partial \boldsymbol{\rho}} g}_{0}+\phi(g) \frac{\partial g}{\partial \boldsymbol{\rho}})
\end{aligned}
$$

whereas an approximation of the Hessian $H=H(\rho)$ is given by

$$
H=\underbrace{\frac{1}{N} \frac{\partial \mathbf{e}^{T}}{\partial \boldsymbol{\rho}} \frac{\partial \mathbf{e}}{\partial \boldsymbol{\rho}}}_{H_{e}}+\alpha \underbrace{\phi^{2}(g)\left(\frac{\partial g}{\partial \boldsymbol{\rho}}\right)^{2}}_{H_{g}} .
$$

From (25) and (26), it follows that finding $\rho^{*}$ in (6) with (7) requires the additional gradients $\partial g / \partial \boldsymbol{\rho}$; the gradients $\partial \mathbf{e} / \partial \boldsymbol{\rho}$ are obtained from the IFT experiments, as explained in Section III.

\section{Constrained Iterative Feedback Tuning Algorithm}

Before deriving the gradients $\partial g / \partial \rho$, consider the resulting constrained IFT algorithm, as shown in Fig. 4.

The algorithm consists of the following basic steps.

1) Conduct three IFT experiments in the context of Fig. 2 with the most recent controller parameters $\rho$.

2) Obtain the gradients $\partial J\left(\rho_{i}\right) / \partial \rho$ and the approximate Hessian $H\left(\rho_{i}\right)$. When coming from step 1, this involves only $\partial J_{e} / \partial \rho$ and $H_{e}\left(\rho_{i}\right)$. When coming from step 4, first, $\partial J_{e} / \partial \rho$ and $H_{e}\left(\rho_{i}\right)$ need to be recomputed, and second, the gradients $\partial J_{g} / \partial \rho$ and the approximate Hessian $H_{g}\left(\rho_{i}\right)$ are required.

3) Using (7), compute the candidate parameter set $\rho_{i+1}$ and update the controller $\mathcal{C}_{f b}=\mathcal{C}_{f b}\left(\rho_{i+1}\right)$

4) On the basis of a nonparametric model, check if $g\left(\boldsymbol{\rho}_{i+1}\right) \leq \epsilon$ with $\epsilon>0$. If satisfied, accept $\boldsymbol{\rho}_{i+1}$ as controller parameter set and either return to step 1 if $i \leq i_{\max }$ or terminate the algorithm otherwise. If not satisfied, proceed with step 2 to find a candidate parameter set $\rho_{i+1}$ that does satisfy $g \leq \epsilon$. However, if $g\left(\boldsymbol{\rho}_{i+1}\right)$ exceeds the user-defined threshold value indicated by $\psi>0$, first the step size $\gamma_{i}$ is reduced so as to damp the convergence process. The motivation for this specific stopping criterion will be given in the following section.

\section{Computing the Gradients $\partial g / \partial \rho$}

In conducting the IFT algorithm in Fig. 4, computing the gradients $\partial g / \partial \rho$ is done upon the violation of the constraint $g$ in (21), i.e., $g>0$. To detect such a constraint violation, a nonparametric model based on frequency response data

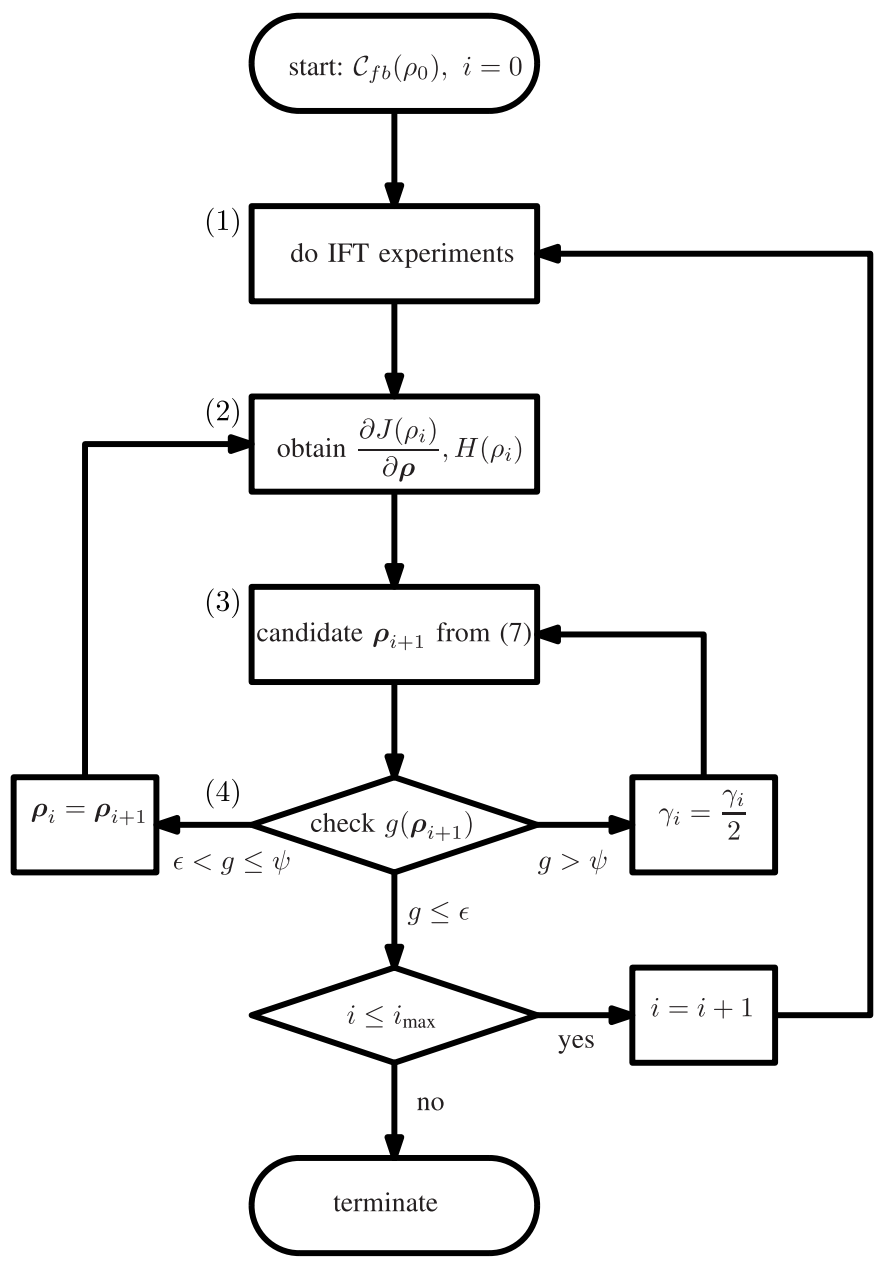

Fig. 4. Overview of the constrained IFT algorithm.

is used for the sensitivity function $\mathcal{S}=\mathcal{S}(\omega, \rho)$ with the candidate parameter set $\boldsymbol{\rho}=\boldsymbol{\rho}_{i+1}$. It is important to realize that no extra experiments are conducted to obtain $\partial g / \partial \rho$. Furthermore, note that frequency response data measurements for motion systems are inexpensive and quickly obtained, but cannot be used directly for controller synthesis, as model-based controller synthesis requires a parametric model. By imposing constraints, machine damage as a result of controller parameters that possibly induce severe violations of the closed-loop frequency-domain specifications will be avoided. In fact, without reasonable assurance that the candidate parameter set $\rho=\rho_{i+1}$ found in step 3 of the IFT algorithm in Fig. 4 avoids such violations, no IFT experiments will be conducted.

To obtain the gradients $\partial g / \partial \rho$, i.e., the constrained part $g$, the center difference scheme is used

$$
\begin{aligned}
\left.\frac{\partial g}{\partial \rho}\right|_{\rho=\rho_{i+1}} & =\left.\frac{\Delta g}{\Delta \rho}\right|_{\rho=\rho_{i+1}}+\mathcal{O}\left(\|h\|^{2}\right) \text { for }\|h\| \rightarrow 0 \\
& \approx\left[\left.\left.\frac{\Delta g}{\Delta \rho(1)}\right|_{\rho=\rho_{i+1}} \ldots \frac{\Delta g}{\Delta \rho(m)}\right|_{\rho=\rho_{i+1}}\right]^{T}
\end{aligned}
$$

i.e., a perturbation method with step sizes $h=\left[h_{1} \ldots h_{m}\right]^{T}$, where $h$ is related to the controller parameters obtained per 


$$
\left.\frac{\Delta g}{\Delta \rho(j)}\right|_{\rho=\rho_{i+1}}=\frac{g\left(\rho(1) \ldots \rho(j)+h_{j} \ldots \rho(m)\right)-g\left(\rho(1) \ldots \rho(j)-h_{j} \ldots \rho(m)\right)}{2 h_{j}}
$$

iteration $i, h_{i}=0.01 \rho_{i}$, and (28), shown at the top of this page

To estimate the effect of the gradient $\partial J_{e} / \partial \rho_{i}$ and approximate Hessian $H_{e}$ coming from the unconstrained part $e$ (and for which we need to recompute the candidate parameter set $\left.\boldsymbol{\rho}=\boldsymbol{\rho}_{i+1}\right)$, the fact is used that

$$
\begin{aligned}
\left.\frac{\partial J_{e}}{\partial \boldsymbol{\rho}}\right|_{\rho=\rho_{i+1}}= & \left.\frac{\partial J_{e}}{\partial \boldsymbol{\rho}}\right|_{\rho=\rho_{i}}+H_{e}\left(\rho_{i}\right) \Delta \boldsymbol{\rho} \\
& +\mathcal{O}\left(\left\|\Delta \boldsymbol{\rho}^{2}\right\|\right) \text { for }\|\Delta \boldsymbol{\rho}\| \rightarrow 0
\end{aligned}
$$

with $\Delta \boldsymbol{\rho}=\boldsymbol{\rho}_{i+1}-\boldsymbol{\rho}_{i}$, which implies

$$
\left.H_{e}\right|_{\rho=\rho_{i+1}}=\left.H_{e}\right|_{\rho=\rho_{i}} .
$$

In [9], the validity of such a parameter approximation is investigated. With (27), (29), and (30) substituted into (25) and (26), a new candidate set of the controller parameters $\rho=\boldsymbol{\rho}_{i+1}$ is computed with (7). In an iterative way, the set $\rho=\rho_{i+1}$ is sought that satisfies $g \leq \epsilon$ with $\epsilon>0$ a constant that can be chosen arbitrary small. Typically, we choose $\epsilon=0.5 \mathrm{~dB}$.

Remark 4: The unconstrained optimization problem in (6) is generally nonconvex, irrespective of adding $J_{g}$ to the original cost function $J_{e}$ in (23). With (7), it is therefore not realistic to expect more than local convergence, i.e., a sufficiently good initial parameter set $\rho_{0}$ is generally required to reach the global minimum; in this paper, $\rho_{0}$ is the result of an industrial (and performance relevant) robust controller tuning process. To ensure convergence, $g \leq \epsilon$ rather than the more generally known stopping criterion $\partial J / \partial \rho \leq \bar{\epsilon}$, with $\bar{\epsilon}$ a threshold, is used. The reason for this is that $g \leq \epsilon$ is a direct measure of the performance objectives in the IFT algorithm of Fig. 4. We thus accept a controlled violation of the frequency-domain robustness specifications by the maximum amount of $\epsilon$. An alternative would be to use a barrier function approach.

Remark 5: In the unconstrained optimization problem of (23), $J_{g}$ is added to $J_{e}$. To scale both parts with respect to each other, the scaling factor $\alpha$ is chosen at $\alpha=10 \mathrm{~nm}^{2}$. This is because $e \sim \mathrm{nm}$ and $g \sim 1$ such that both parts $J_{e}$ and $J_{g}$ become equally weighted in (23). Similarly, the optimization window, i.e., the sampled data interval used to define $J_{e}$ in (23), should be chosen with the application specifics in mind. For the stage control case, the data sampled signals e have $N=500$ samples with 250 samples in the acceleration phase and 250 samples in the scanning phase of constant velocity (see [13] for more details on the choice of optimization window).

Remark 6: An important aspect in the IFT algorithm of Fig. 4 is the choice of the iteration factor $\gamma_{i}$ in (7). The strategy that is being followed is to start with $\gamma_{0}=1$ [recall step 1 in Fig. 4], and then do step refinement depending on the amount of violation that is encountered with respect to the constant $\psi>0$ [20]. Typically, we choose $\psi$ that is a measure of violation at $\psi=1 \mathrm{~dB}$. Constraint violations $g>\psi$ will induce step refinement. Constraint violations $\epsilon<g \leq \psi$ do not induce step refinement so as to avoid too slow convergence rates. Constraint violations $g \leq \epsilon$ (in the case that $\left.i \leq i_{\max }\right)$ induce a reinitialization of the step size to $\gamma_{i}=1$. A representative sequence for the step size $\gamma_{i}$, such as encountered in the experiments during five iterations, is given by $\{1,0.25,0.25,0.0625,0.001953125\}$. Note that reducing $\gamma_{i}$ comes with the risk of effectively halting the optimization. In reducing this risk, one could decide to choose a larger $\psi$.

\section{Measurement Results}

In this section, the measurement results are presented that are obtained with constrained IFT on a wafer scanner. Since constrained IFT does not require a parametric plant model, it is more convenient to apply constrained IFT directly to the wafer stage system rather than doing simulation studies first. For the experiments, the motion control structure from Fig. 2 is used including the auxiliary input $w$. In presenting the results, this section is divided into four parts: 1) parameter convergence; 2) optimized controller settings; 3 ) robust stability aspects; and 4) time-domain performance.

\section{A. Parameter Convergence}

Given the structure of the wafer stage feedback controller $\mathcal{C}_{f b}=\mathcal{C}_{f b}(\rho)$ in (1), constrained IFT refers to tuning the PIDcontroller parameters $k_{p}, \omega_{i}$, and $\omega_{d}$ (see also [25], [26], [41]), and the additional notch filter frequencies $\omega_{z, i}, \omega_{p, i}$, or

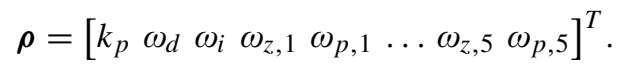

Note that machine-in-the-loop optimization of the notch filters is relevant for the following reason. Limitations in current machine performance due to unknown variations from machine to machine in terms of disturbances or frequency characteristics (resonances) are likely to be overcome by machine-dedicated tunings that properly address these variations.

Remark 7: In optimizing the feedback controller parameters $\rho$ in $\mathcal{C}_{f b}=\mathcal{C}_{f b}(\rho)$ with constrained IFT, the zero and pole damping coefficients $\zeta_{z, i}, \zeta_{p, i}$ are fixed (see also Table I in Section II). This rather arbitrary choice yields a reduced number of parameters to be optimized, which reduces numerical complexity, but (potentially) comes at the cost of performance. It is clear that such design choices strongly relate to the (performance) specifics of the application at hand. The low-pass filter $\mathcal{C}_{\mathrm{LP}}$ in (1) is fixed as to assure high-frequency rolloff.

The results of the IFT algorithm in terms of parameter convergence are shown in Fig. 5. Given an identical set of controller parameters $\rho_{0}$ at iteration number $i=0$, it can be observed that convergence occurs in basically five iteration steps. The differences between the initial and final parameter sets obtained with constrained IFT are mainly caused by the variation in disturbances and (plant) dynamics from machine 

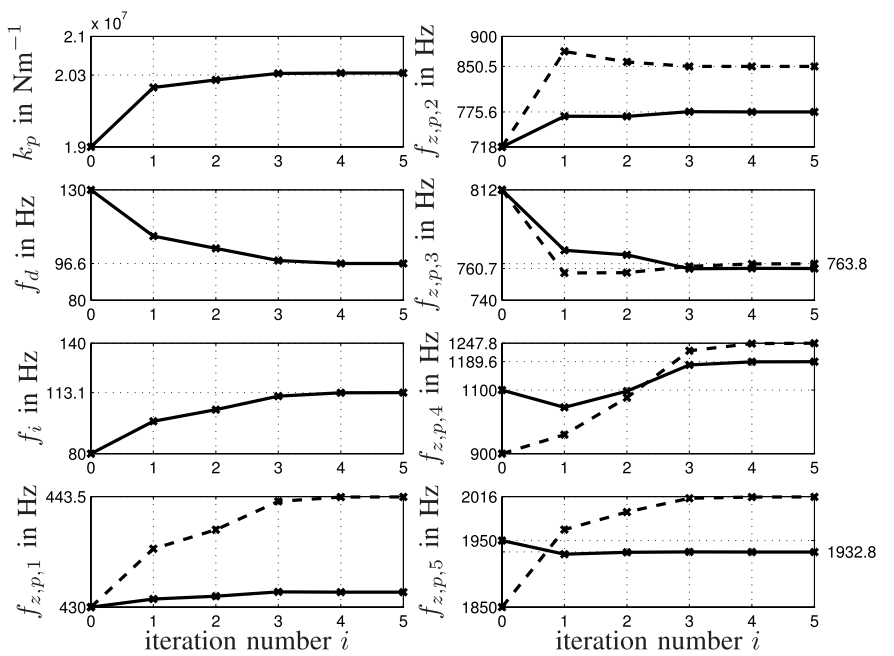

iteration number $i$

Fig. 5. Measured parameter convergence diagram. For the notch filters $\mathcal{N}_{i}$, convergence of the zero frequencies $f_{z, i}=\omega_{z, i} / 2 \pi$ is indicated by the solid curves, while convergence of the pole frequencies $f_{p, i}=\omega_{p, i} / 2 \pi$ is indicated by the dashed curves. $\gamma_{0}=1$ and $\epsilon=0.5 \mathrm{~dB}$.
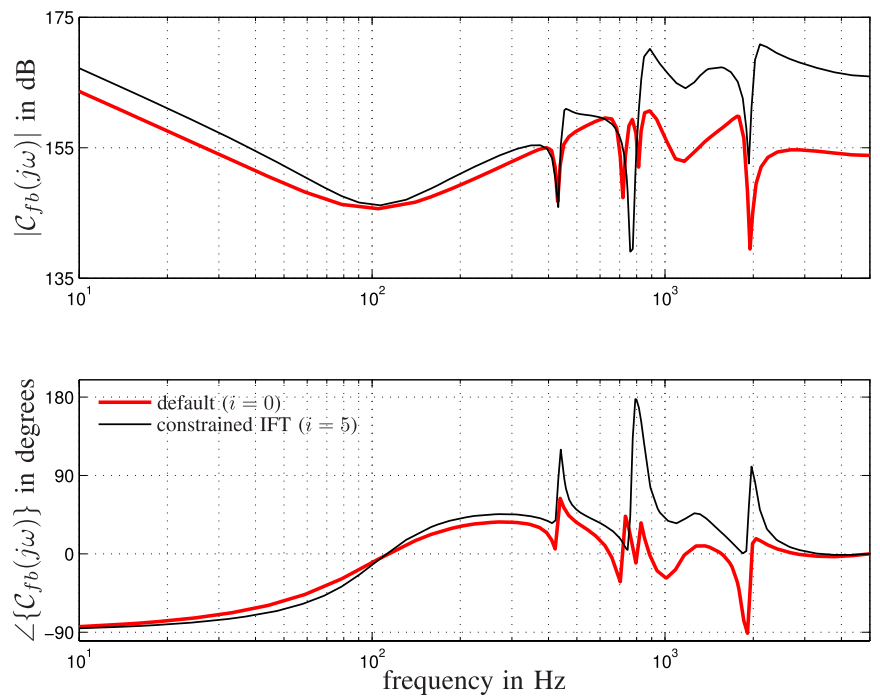

Fig. 6. Bode diagrams of the controllers before $(i=0)$ and after $(i=5)$ optimization.

to machine, hence the need for a machine-dedicated tuning of the notch filters. Note the subtle differences (for example) in notch filter $\mathcal{N}_{1}$, which becomes a skew notch filter in order to obtain more disturbance suppression around $400 \mathrm{~Hz}$, while notch filter $\mathcal{N}_{3}$ demonstrates lowering of the notch frequency, but leaving the notch shape unchanged so as to avoid noise amplification around $760 \mathrm{~Hz}$. Both frequencies relate to turbo pumps that through excitation of the metrology frame disturb the interferometer measurement system, i.e., disturbances that corrupt the plant output (Fig. 2). Interestingly, Fig. 5 shows that convergence in the parameter space is nonmonotonic. This indicates that $J$ is probably nonquadratic in the parameters, necessitating several iterations of (7).

\section{B. Optimized Controller Settings}

Given the controller parameter sets $\rho_{0}$ and $\rho_{5}$ found in Fig. 5 at $i=0$ and $i=5$, respectively, Fig. 6 shows the
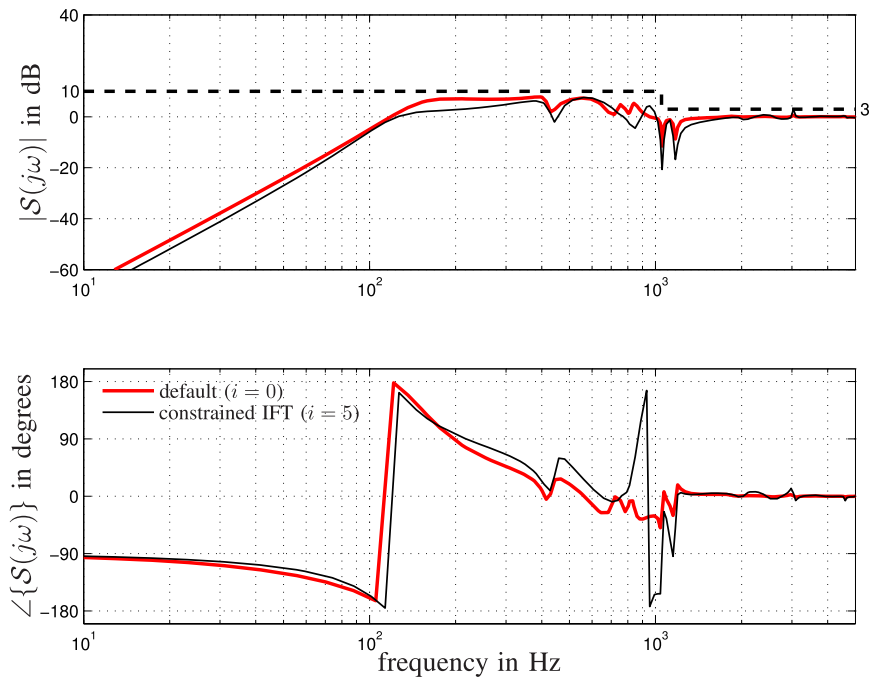

Fig. 7. Bode diagrams of the sensitivity function $\mathcal{S}$ based on measured data before $(i=0)$ and after $(i=5)$ constraint IFT optimization. $\mathcal{S}_{b}=9.5 \mathrm{~dB}$ on

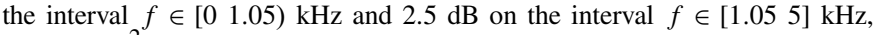
$\alpha=10 \mathrm{~nm}^{2}, \gamma_{0}=1$, and $\epsilon=0.5 \mathrm{~dB}$.

Bode diagrams of the resulting controllers $\mathcal{C}_{f b}$. From Fig. 5, it can be observed that constrained IFT induces $\approx 3.5 \mathrm{~dB}$ extra low-frequency disturbance rejection below $30 \mathrm{~Hz}$, but at the cost of $\approx 12-\mathrm{dB}$ high-frequency noise amplification around $5 \mathrm{kHz}$. From the perspective of error minimization, this result is clear. The spectral contents of the error signals are (relatively) small at the high-frequency interval, thereby justifying the amplifications. The formulation of a performance-relevant cost function can therefore be observed as the crucial step in the control design [32]. Apart from the more subtle differences in the notch filter design beyond $800 \mathrm{~Hz}$, a $90^{\circ}$ phase lead is induced with respect to the default parameter set $\rho_{0}$. This involves the first plant resonance around $1 \mathrm{kHz}$ (recall Fig. 3), and will be discussed in the following section.

\section{Robust Stability Aspects}

The comparison of the default parameter set $\rho_{0}$ in Fig. 5 with the constrained IFT parameter set $\rho_{5}$ in terms of the closed-loop sensitivity function $\mathcal{S}$ in (11) is shown in Fig. 7. It can be observed that with constrained IFT, the final parameter set $\rho_{5}$ induces improved low-frequency performance (below $80 \mathrm{~Hz}$ but also between 110 and $500 \mathrm{~Hz}$ ) while satisfying the indicated amplitude constraints $\mathcal{S}_{b}(\omega)+\epsilon$ (dashed curve).

Remark 8: Note that using a weighted modulus margin, robust stability is obtained against inverse multiplicative uncertainties. Moreover, the nonparametric weighting is different from the standard approaches used in robust control.

Remark 9: As to avoid machine damage, IFT (without constraints) is not applied to the machine. The reason for this is shown in Fig. 8, which contains the simulation results only: IFT induces unacceptable amplifications at high frequencies. With constrained IFT, it can be observed that the amplitude constraints are merely touched upon at distinct frequency points. The latter observation could favor the introduction of frequency-varying weights, as done in [14]. 

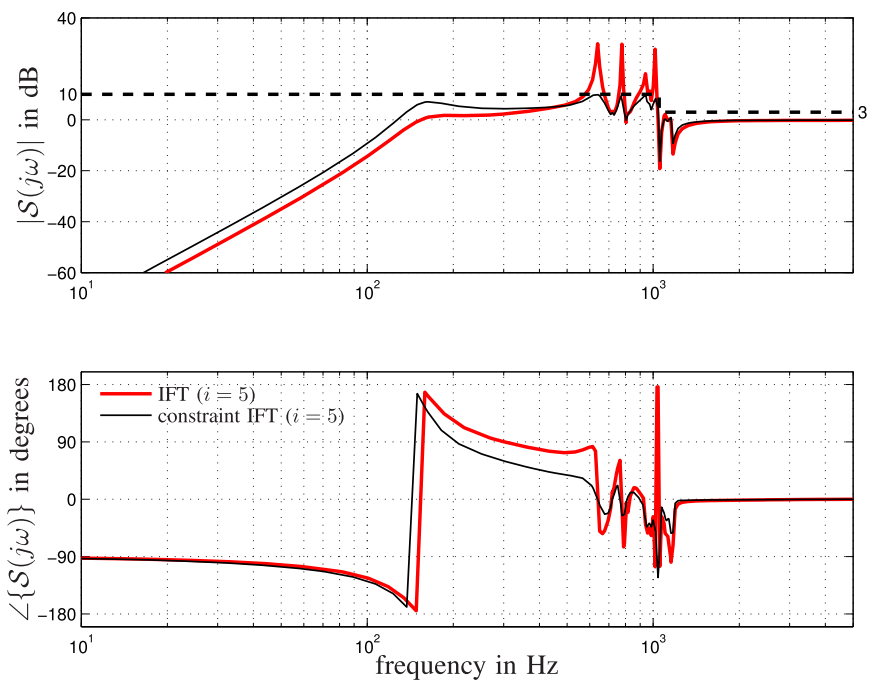

Fig. 8. Bode diagrams of the sensitivity function $\mathcal{S}$ based on simulated data with $\operatorname{IFT}(i=5)$, i.e., no constraints, and with constraint IFT $(i=5)$. $\mathcal{S}_{b}=9.5 \mathrm{~dB}$ on the interval $f \in\left[\begin{array}{ll}0 & 1.05\end{array}\right] \mathrm{kHz}$ and $2.5 \mathrm{~dB}$ on the interval $f \in[1.055] \mathrm{kHz}, \alpha=10 \mathrm{~nm}^{2}, \gamma_{0}=1$, and $\epsilon=0.5 \mathrm{~dB}$.

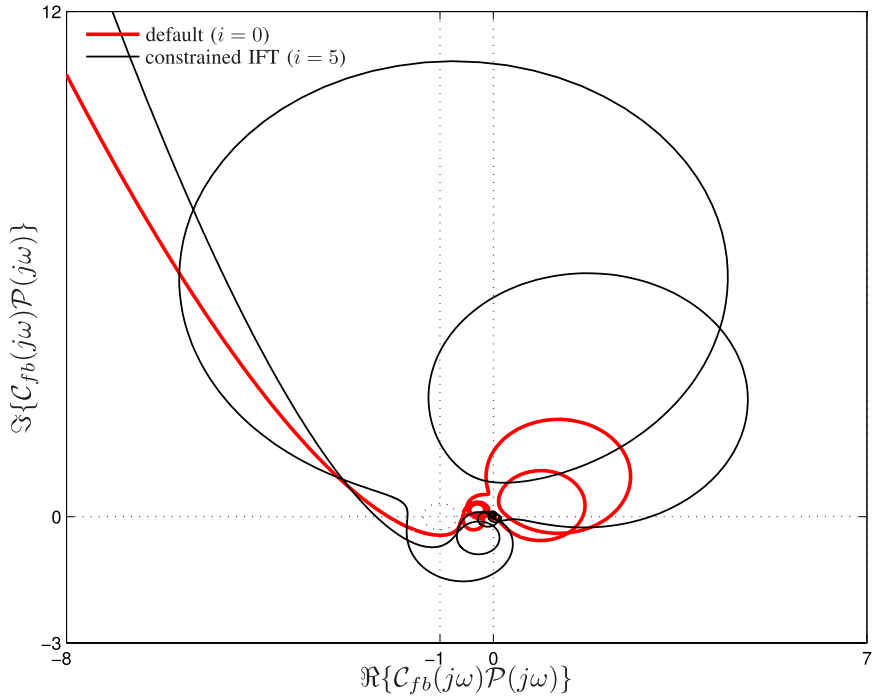

Fig. 9. Nyquist plots of the open-loop frequency response functions before $(i=0)$ and after $(i=5)$ optimization.

Robust stability properties by constraining the sensitivity (amplitude) characteristics also follow from the Nyquist plot evaluation in Fig. 9. In Fig. 9, it can be observed that the modulus margin of $10 \mathrm{~dB}$ is met (either before or after optimization) by the proper encirclement of the disk with a radius of $\approx 0.32$ around the point -1 . Moreover, the earlier mentioned phase lead of $90^{\circ}$, which is induced by constrained IFT around the plant resonance frequency of $1 \mathrm{kHz}$, allows for amplification around this frequency (Fig. 7) and leads to $|\mathcal{S}(\omega=2 \pi 1000)| \approx-20 \mathrm{~dB}$.

Remark 10: The optimized controller obtained through constrained IFT clearly outperforms the default controller. This, however, is partly the result of different optimization objectives. Namely, the default controller tuning is the result of a frequency-domain (and loop-shaping-based) optimization process in which frequency response data are used from
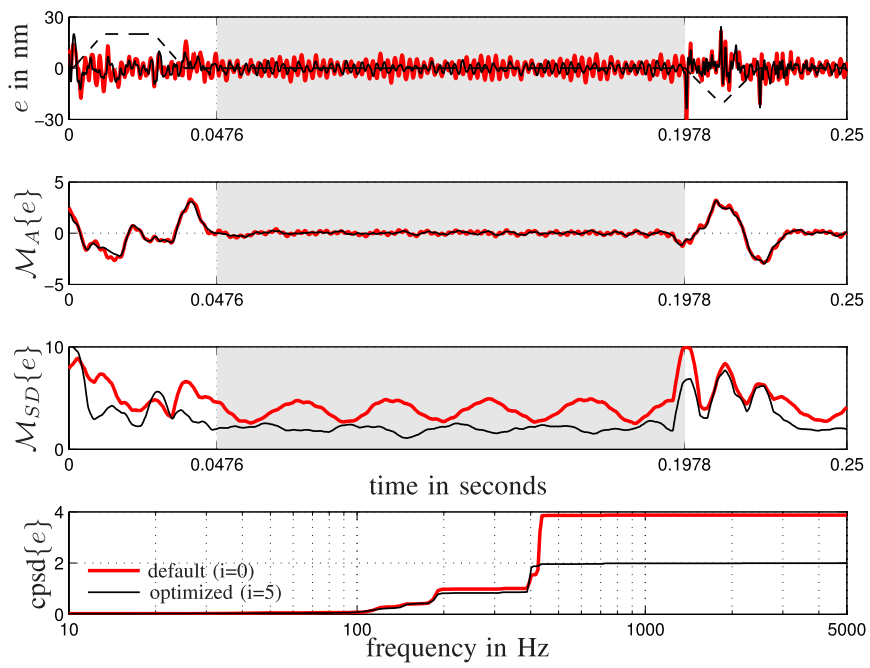

Fig. 10. Time-series representation of experimental data at iteration $i=0$ and iteration $i=5$ together with the results from cumulative power spectral density analysis. The scaled acceleration setpoint profile is indicated with the dashed lines. The scanning performance window is indicated in gray.

several machines. As such, a controller tuning is obtained that is robust against a certain degree of machine variation. But this tuning is not very likely to achieve optimal performance when applied to specific machines, in particular, when, in the tuning process, machine-specific disturbances have not been taken into account.

\section{Time-Domain Performance}

The effect of constrained IFT in terms of time-domain performance is shown in Fig. 10. Performance is evaluated for representative point-to-point scanning motion at a corner part of the wafer (see the dashed curves for the scaled acceleration setpoint profile). In the top part of Fig. 10, unfiltered error responses $e$ are shown before $(i=0)$ and after $(i=5)$ optimization. The middle part of Fig. 10 shows these responses after either using a moving average filter operation $\mathcal{M}_{A}$-as a measure for machine overlay-or a moving standard deviation filter operation $\mathcal{M}_{\mathrm{SD}}$-as a measure for machine imaging (recall the explanation of (4)). The bottom part of Fig. 10 shows a cumulative power spectral density analysis of the error responses $e$. From Fig. 10, it is clear that in terms of error responses, constrained IFT mainly contributes to the removal of the frequency contributions around $400 \mathrm{~Hz}$, which are caused by the earlier mentioned exogenous disturbances from turbo pumps. These disturbances mainly induce $\mathcal{M}_{\mathrm{SD}}$-filtered errors. This machine-specific advantage was not fully known in advance, and therefore could not be properly anticipated for in the (manual) loop-shaping tunings. This confirms the potential benefit of IFT to account for actual disturbance spectra in a control-relevant manner.

\section{CONCLUSION}

In this paper, a new approach for constrained IFT has been presented and experimentally demonstrated on the highprecision stage systems of a lithography machine. The IFT 
algorithm is extended with a frequency-domain penalty to penalize violations of the frequency-domain specifications imposed on the closed-loop sensitivity function. Using a nonparametric model, an adaptation is made to the candidate parameter set obtained from IFT. The prediction involves a straightforward Taylor series expansion part using the resulting unbiased gradient estimates. In addition, it involves a part that uses the perturbation method to obtain the gradients with respect to the penalty. With the adaptation, a parameter set is obtained that from a model point of view meets the frequencydomain specifications. As a result, constrained IFT is done under the assurance that: 1) frequency-domain specifications are satisfied or 2) violations of the specifications stay within controllable levels. For both the PID and notch filter controller parameters of a generally more advanced controller structure, it is demonstrated that machine-specific performance, which is clearly disturbance related, can be obtained with constrained IFT. In time domain, constrained IFT is used to create the machine-specific fine-tunings of the feedback control design in addition to the nominal loop-shaping tuning process that is done in frequency domain. The ongoing work involves the MIMO case, effective constraint formulation, and the development of efficient algorithms.

\section{ACKNOWLEDGMENT}

The authors would like to thank Dr. D. Kostić and Dr. B. de Jager for their contributions in an early stage of this research.

\section{REFERENCES}

[1] A. Al Mamun, W. Y. Ho, W. E. Wang, and T. H. Lee, "Iterative feedback tuning (IFT) of hard disk drive head positioning servomechanism," in Proc. 33rd Annu. Conf. IEEE Ind. Electron. Soc., Taipei, Taiwan, Nov. 2007, pp. 769-774.

[2] A. S. Bazanella, L. Campestrini, and D. Eckhard, Data-Driven Controller Design: The $\mathcal{H}_{2}$ Approach. New York, NY, USA: Springer-Verlag, 2012.

[3] F. Boeren, T. Oomen, and M. Steinbuch, "Iterative motion feedforward tuning: A data-driven approach based on instrumental variable identification," Control Eng. Pract., vol. 37, pp. 11-19, 2015.

[4] M. Boerlage, M. Steinbuch, P. Lambrechts, and M. van de Wal, "Modelbased feedforward for motion systems," in Proc. IEEE Conf. Control Appl., Jun. 2003, pp. 1158-1163.

[5] F. De Bruyne and L. C. Kammer, "Iterative feedback tuning with guaranteed stability," in Proc. Amer. Control Conf., San Diego, CA, USA, 1999, pp. 3317-3321.

[6] H. Butler, "Position control in lithographic equipment [Applications of Control]," IEEE Control Syst. Mag., vol. 31, no. 5, pp. 28-47, Oct. 2011.

[7] M. C. Campi, A. Lecchini, and S. M. Savaresi, "Virtual reference feedback tuning: A direct method for the design of feedback controllers," Automatica, vol. 38, no. 8, pp. 1337-1346, 2002.

[8] S. Garatti, M. C. Campi, and S. Bittanti, "Iterative robust control: Speeding up improvement through iterations," Syst. Control Lett., vol. 59, no. 2, pp. 139-146, 2010.

[9] M. Gevers, "A decade of progress in iterative process control design: From theory to practice," J. Process Control, vol. 12, no. 4, pp. 519-531, 2002.

[10] S. Gunnarsson, O. Rousseaux, and V. Collignon, "Iterative feedback tuning applied to robot joint controllers," in Proc. 14th IFAC World Congr., Beijing, China, 1999, pp. 451-456.

[11] K. Hamamoto, T. Fukuda, and T. Sugie, "Iterative feedback tuning of controllers for a two-mass-spring system with friction," Control Eng. Pract., vol. 11, no. 9, pp. 1061-1068, 2003.

[12] A. Hansson, K. El-Awady, and B. Wahlberg, "A primal-dual interiorpoint method for iterative feedback tuning," in Proc. IFAC World Congr., Beijing, China, 1998, pp. 36-41.
[13] M. F. Heertjes, D. Hennekens, and M. Steinbuch, "MIMO feed-forward design in wafer scanners using a gradient approximation-based algorithm," Control Eng. Pract., vol. 18, no. 5, pp. 495-506, 2010.

[14] M. F. Heertjes, M. Galluzzo, and L. Kuindersma, "Robust data-driven control for the stage synchronization problem," in Proc. 19th IFAC World Congr., Cape Town, South Africa, 2014, pp. 1134-1139.

[15] H. Hjalmarsson, S. Gunnarsson, and M. Gevers, "A convergent iterative restricted complexity control design scheme," in Proc. 33rd IEEE Conf. Decision Control, Lake Buena Vista, FL, USA, Dec. 1994, pp. $1735-1740$.

[16] H. Hjalmarsson and T. Birkeland, "Iterative feedback tuning of linear time-invariant MIMO systems," in Proc. 37th IEEE Conf. Decision Control, Tampa, FL, USA, Dec. 1998, pp. 3893-3898.

[17] H. Hjalmarsson, "Efficient tuning of linear multivariable controllers using iterative feedback tuning," Int. J. Adapt. Control Signal Process., vol. 13, no. 7, pp. 553-572, 1999.

[18] H. Hjalmarsson, "Iterative feedback tuning-An overview," J. Adapt. Control Signal Process., vol. 16, no. 5, pp. 373-395, 2002.

[19] H. Hjalmarsson, "From experiment design to closed-loop control," Automatica, vol. 41, no. 3, pp. 393-438, 2005.

[20] J. K. Huusom, N. K. Poulsen, and S. B. Jørgensen, "Improving convergence of iterative feedback tuning," J. Process Control, vol. 19, no. 4, pp. 570-578, 2009.

[21] J. K. Huusom, H. Hjalmarsson, N. K. Poulsen, and S. B. Jørgensen, "A design algorithm using external perturbation to improve iterative feedback tuning convergence," Automatica, vol. 47, no. 12, pp. 2665-2670, 2011

[22] S. Kissling, P. Blanc, P. Myszkorowski, and I. Vaclavik, "Application of iterative feedback tuning (IFT) to speed and position control of a servo drive," Control Eng. Pract., vol. 17, no. 7, pp. 834-840, 2009.

[23] D. Kostić, "Data-driven robot motion control design," Ph.D. dissertation, Dept. Mech. Eng., Eindhoven Univ. Technol., Eindhoven, The Netherlands, 2004.

[24] P. Lambrechts, M. Boerlage, and M. Steinbuch, "Trajectory planning and feedforward design for high performance motion systems," in Proc. Amer. Control Conf., Boston, MA, USA, 2004, pp. 4637-4642.

[25] O. Lequin, "Optimal closed loop PID tuning in the process industry with the iterative feedback tuning scheme," in Proc. 4th Eur. Control Conf., Brussels, Belgium, 1997, paper TH-A-H6.

[26] O. Lequin, M. Gevers, M. Mossberg, E. Bosmans, and L. Triest, "Iterative feedback tuning of PID parameters: Comparison with classical tuning rules," Control Eng. Pract., vol. 11, no. 9, pp. 1023-1033, 2003.

[27] H. J. Levinson, Principles of Lithography, 3rd ed. Bellingham, WA, USA: SPIE, 2010.

[28] D. Liu, A. J. McDaid, K. C. Aw, and S. Q. Xie, "Position control of an ionic polymer metal composite actuated rotary joint using iterative feedback tuning," Mechatronics, vol. 21, no. 1, pp. 315-328, 2011.

[29] K. Mahathanakiet, M. A. Johnson, A. Sanchez, and M. J. Wade, "Iterative feedback tuning and an application to a wastewater treatment plant," in Proc. 4th Asian Control Conf., Singapore, 2002, pp. 256-261.

[30] S. H. Van der Meulen, R. L. Tousain, and O. H. Bosgra, "Fixed structure feedforward controller design exploiting iterative trials: Application to a wafer stage and a desktop printer," J. Dyn. Syst., Meas., Control, vol. 130 , no. 5 , p. 051006,2008

[31] R. H. Middleton and G. C. Goodwin, "Improved finite word length characteristics in digital control using delta operators," IEEE Trans. Autom. Control, vol. 31, no. 11, pp. 1015-1021, Nov. 1986.

[32] M. Mossberg, "Controller tuning via minimization of time weighted absolute error," in Proc. Amer. Control Conf., Boston, MA, USA, 2004, pp. 2740-2744.

[33] T. Oomen, R. van Herpen, S. Quist, M. van de Wal, O. H. Bosgra, and M. Steinbuch, "Connecting system identification and robust control for next-generation motion control of a wafer stage," IEEE Trans. Control Syst. Technol., vol. 22, no. 1, pp. 102-118, Jan. 2014.

[34] H. Prochazka, M. Gevers, B. D. O. Anderson, and C. Ferrera, "Iterative feedback tuning for robust controller design and optimization," in Proc. 44th IEEE Conf. Decision Control, Eur. Control Conf., Seville, Spain, Dec. 2005, pp. 3602-3607.

[35] M.-B. Radac, R.-E. Precup, S. Preitl, and C.-A. Dragos, "Iterative feedback tuning in MIMO systems. Signal processing and application," in Proc. 5th Int. Symp. Appl. Comput. Intell. Inform., Timişoara, Romania, 2009, pp. 77-82.

[36] Z. P. Rico, A. Lecchini-Visintini, and R. Q. Quiroga, "Iterative feedback tuning for the joint controllers of a 7-DOF whole arm manipulator," in Proc. IEEE 51st Annu. Conf. Decision Control, Maui, HI, USA, Dec. 2012, pp. 544-549. 
[37] D. Rupp and L. Guzzella, "Iterative tuning of internal model controllers with application to air/fuel ratio control," IEEE Trans. Control Syst. Technol., vol. 18, no. 1, pp. 177-184, Jan. 2010.

[38] M. G. Safonov and T.-C. Tsao, "The unfalsified control concept and learning," IEEE Trans. Autom. Control, vol. 42, no. 6, pp. 843-847, Jun. 1997.

[39] J. Sjöberg et al., "Iterative controller optimization for nonlinear systems," Control Eng. Pract., vol. 11, no. 9, pp. 1079-1086, 2003.

[40] G. Solari and M. Gevers, "Unbiased estimation of the Hessian for iterative feedback tuning (IFT)," in Proc. 43rd IEEE Conf. Decision Control, Paradise Island, Bahamas, Dec. 2004, pp. 1759-1760.

[41] B. J. C. H. Van der Velden, T. Oomen, and M. F. Heertjes, "Constrained iterative feedback tuning for robust high-precision motion control," in Proc. 19th IFAC World Congr., Cape Town, South Africa, 2014, pp. 4915-4920.

[42] S. Veres and H. Hjalmarsson, "Tuning for robustness and performance using iterative feedback tuning," in Proc. 41st IEEE Conf. Decision Control, Las Vegas, NV, USA, Dec. 2002, pp. 4682-4687.

[43] G. Vinnicombe, "Frequency domain uncertainty and the graph topology," IEEE Trans. Autom. Control, vol. 38, no. 9, pp. 1371-1383, Sep. 1993.

[44] M. van de Wal, G. van Baars, F. B. Sperling, and O. H. Bosgra, "Multivariable $\mathcal{H}_{\infty} / \mu$ feedback control design for high-precision wafer stage motion," Control Eng. Pract., vol. 10, no. 7, pp. 739-755, 2002.

[45] P.-H. Yang and S.-L. Koo, "Control systems and methods applying iterative feedback tuning for feed-forward and synchronization control of microlithography stages and the like," U.S. Patent US 8451431 B2, May 28, 2013.

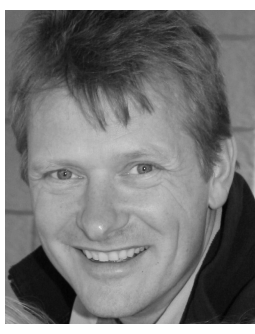

Marcel F. Heertjes received the M.Sc. and Ph.D. degrees in mechanical engineering from the Department of Mechanical Engineering, Eindhoven University of Technology, Eindhoven, The Netherlands, in 1995 and 1999, respectively.

$\mathrm{He}$ was with the Philips Centre for Industrial Technology, Eindhoven, from 2000 to 2006. In 2007, he joined ASML, Veldhoven, The Netherlands, as a Mechatronics Development Engineer, where he is currently a Principle Engineer. He was with the Control System Technology and Dynamics and Control Groups, Department of Mechanical Engineering, Eindhoven University of Technology, where he is an Associate Professor. His current research interests include the control of industrial motion systems with special attention to nonlinear control, feedforward and learning control, and machine-in-the-loop controller optimization and self-tuning.

Dr. Heertjes served as a Guest Editor of the Special Issue on Performance of Nonlinear Control Systems in the International Journal of Robust and Nonlinear Control in 2013, and the Special Issue on Control of High-Precision Motion Systems in IFAC Mechatronics in 2014.

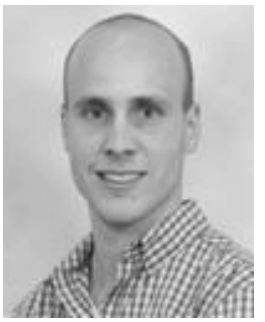

Bart Van der Velden received the M.Sc. degree from the Eindhoven University of Technology, Eindhoven, The Netherlands, in 2014. His thesis was on the subject of constrained iterative feedback tuning applied to high-precision motion stages, which was done in co-operation with ASML, Veldhoven, The Netherlands.

$\mathrm{He}$ is currently a Support Engineer with ASML, where his main responsibility is to provide handson trouble shooting support in lithography systems during production and installation. His expertise is in mechatronics and dynamics.

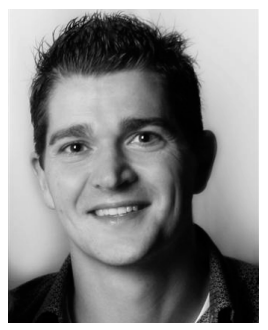

Tom Oomen received the M.Sc. (cum laude) and $\mathrm{Ph} . \mathrm{D}$. degrees from the Eindhoven University of Technology, Eindhoven, The Netherlands, in 2005 and 2010 , respectively.

He held visiting positions with the KTH Royal Institute of Technology, Stockholm, Sweden, and The University of Newcastle, Callaghan, NSW, Australia. $\mathrm{He}$ is currently an Assistant Professor with the Department of Mechanical Engineering, Eindhoven University of Technology. His current research interests include system identification, robust control, and learning control, with applications in mechatronic systems.

Dr. Oomen was a recipient of the Corus Young Talent Graduation Award in 2005. He has served on the IEEE Conference Editorial Board as an Associate Editor, and a Guest Editor of IFAC Mechatronics. 